\title{
Modelling and thermal simulation of absorber-free quasi-simultaneous laser welding of transparent plastics
}

\author{
Nam-Phong Nguyen ${ }^{1}\left[\right.$ D $\cdot$ Stefan Behrens ${ }^{1} \cdot$ Maximilian Brosda $^{1} \cdot$ Alexander Olowinsky $^{1} \cdot$ Arnold Gillner $^{2}$
}

Received: 9 December 2019 / Accepted: 23 July 2020 / Published online: 25 August 2020

(C) The Author(s) 2020

\begin{abstract}
The growing demands on the quality of plastic components and the trend towards miniaturisation are posing a great challenge on plastics processing technology. As many complex components can no longer be manufactured in a single step, joining processes such as laser transmission welding are gaining in importance. In classic laser transmission welding, the joining partners have different optical properties. The upper joining partner is transparent in the laser wavelength range, whilst the lower partner is absorbent due to the addition of absorber materials. In medical and biotechnological applications, the addition of absorber materials is often undesirable due to strict biocompatibility requirements. If, on the other hand, radiation sources are used which emit radiation in the area of the natural absorption of the plastic $(\lambda=1600-2000 \mathrm{~nm})$, untreated transparent plastics can also be welded. In this work, a theoretical model will be presented to calculate the temperature distribution and progression during quasi-simultaneous welding using a thulium fibre laser $(\lambda=1940 \mathrm{~nm})$. A sensitivity analysis is carried out to investigate the influence of different parameters on the heat affected zone (HAZ). The simulated HAZ is then compared with the HAZ from the experimental work.
\end{abstract}

Keywords Plastic joining $\cdot$ Transparent polymers $\cdot$ Thermal simulation $\cdot$ Laser transmission welding

\section{Introduction}

The growing demands on the quality of plastic components and the trend towards miniaturisation are posing a great challenge on plastics processing technology. As many complex components can no longer be manufactured in a single step, joining processes such as laser transmission welding are gaining in importance [1]. For example, microfluidic components such as lab-on-a-chip devices combine the functionalities of analytical instruments in a small chip. The higher portability allows these tests to be performed on site. The challenge here is the media-tight encapsulation of the narrow and complex channel structures with channel diameters less than $300 \mu \mathrm{m}$.

Recommended for publication by Commission XVI - Polymer Joining and Adhesive Technology

Nam-Phong Nguyen

phong.nguyen@ilt.fraunhofer.de

1 Fraunhofer Institute for Laser Technology ILT, Steinbachstr. 15, 52074 Aachen, Germany

2 Chair for Laser Technology LLT, Steinbachstr. 15, 52074 Aachen, Germany
One promising approach is laser transmission welding. The temporal and spatial defined and contactless energy input leads to a precise welding process at low thermal and mechanical stress [2, 3]. In classic laser transmission welding, beam sources are used which emit radiation in the transparent wavelength range of most polymers $(\lambda=800-1100 \mathrm{~nm})$. Therefore, the modification of the optical components is necessary to melt the joining partners. Generally, the joining partners are welded as an overlap joint. A clamping device enables an exact and reproducible positioning of the joining partners. Furthermore, a thermal contact is achieved by applying a joining pressure. During the welding process, the incident laser beam is transmitted by the upper transparent joining partner and absorbed at the surface of the lower absorbent joining partner. The absorbing properties of the lower joining partner are generated by using an absorber material such as carbon black. The electromagnetic irradiation energy is converted into heat which leads to melting of both joining partners in the joining zone. A welding seam is then generated as soon as the melt solidifies $[3,4]$. One important factor for the achievement of a high and reproducible welding seam quality is the amount of absorber material. Here, an exact dosage is decisive as fluctuations of the absorber amount lead to an uneven absorption and therefore to defects of the welding seam [5]. On 
top of that, the black colouring of the component is inadmissible in areas such as biotechnology or medical engineering [6]. Alternatively, it is possible to use near-infrared absorbers which do not or only slightly affect the colour of the component. As the price is significantly higher, this has a huge impact on high volumetric compounds [7].

By using new beam sources which emit radiation in the area of the polymeric absorption bands $(\lambda=1600-2000 \mathrm{~nm})$, it is nowadays possible to join transparent polymers without the use of absorbers. The melting is achieved by the excitation of the first harmonic of various bonds such as methyl and methylene groups. Promising application fields apart from the encapsulation of microfluidics is the sealing of polymer films, e.g. food packaging or sensitive electronic components such as OLEDs [7, 8].

With the help of thermal simulations, the temporally and spatially resolved temperature distribution and progression can be determined inside the material. In case of classic laser transmission welding, many works are available based on a finite element method (FEM). For instance, Kreimeier calculated the residual stress inside the welding seam due to the thermal expansion and shrinkage [9]. Other works deal with the influence of absorber materials such as carbon black or indium tin oxide on the temperature field and the dimensions of the HAZ $[1,5,10]$. In order to simulate the welding process of semicrystalline polymers, the light scattering effects have to be taken into account. For example, this can be either done experimentally by measuring the intensity profile and beam broadening using camera-based systems [11], by calculating the scattering behaviour with the help of Monte Carlo simulations $[1,12]$ or by using a laser-line scan technique as proposed in [13-15].

In case of absorber-free laser transmission welding, a quasi-simultaneous irradiation leads to a reduction of the HAZ dimensions compared with contour welding. This leads to a lower thermal stress and reduces the risks of a formation of cracks and distortions $[6,16]$. In this work, a theoretical model will be presented to calculate the temperature distribution in absorber-free quasi-simultaneous welding using the FEM. First, the modelling of the heat source will be presented. The temperature progression as well as the distribution will be investigated during the welding process. In order to determine the influencing factors on the HAZ, a sensitivity analysis is carried out. Furthermore, the simulated results will be compared with the HAZ of the real welding experiments.

\section{Thermal simulation of absorber-free laser transmission welding}

\subsection{Fundamentals}

The transient temperature field in plastics can be described by Fourier's differential equation [1]:
$\rho(T) * c_{P}(T) *\left(\frac{\partial T}{\partial t}+\vec{v} * \nabla T\right)=\nabla *(k * \nabla T)+Q$

where $\rho$ is the density, $c_{P}$ is the heat capacity, $k$ is the heat conductivity, and $Q$ is the heat source.

The heat generated by the laser radiation is indicated by the internal heat source $\mathrm{Q}$. The convective heat flux is described by the term $\nabla *(k * \nabla T)$, the diffusive heat flux by $\vec{v} * \nabla T$. If the movement of the laser beam is integrated into the heat source, the heat equation is simplified to:

$\rho(T) * c_{P}(T) *\left(\frac{\partial T}{\partial t}\right)=\nabla *(k * \nabla T)+Q$

The heat source $\mathrm{Q}$ is determined by the absorbed laser beam intensity during propagation through the material. In case that no scattering occurs inside the material (such as in amorphous polymers), the heat source can be described by the absorbed laser intensity [9]:

$Q=\alpha^{*} I(x, y, z, t, \alpha)$

where $\alpha$ is the absorption coefficient. The intensity profile of an ideal laser beam has a Gaussian distribution. It can be described as:

$I(r, z)=I_{0}(z) * e^{-\frac{2 r 2}{w(z) 2}}$

$\mathrm{I}_{0}(\mathrm{z})$ is defined as:

$I_{0}(z)=I_{0} *\left(\frac{w_{0}}{w(z)}\right)^{2}$

where $w_{0}$ is the focal beam diameter. The beam radius as a function of the $\mathrm{z}$-coordinate is determined by:

$w(z)=w_{0} * \sqrt{1+\left(\frac{z}{z_{R}}\right)^{2}}$

where $z_{R}$ is the Rayleigh length and $w_{o}$ is the focal diameter. The intensity is defined as power per area. Consequently, the laser power $P_{\mathrm{L}}$ can be calculated by the integral over the radial intensity distribution.

$P_{L}=\int_{\varphi=0}^{2 \pi} \int_{r=0}^{\infty} I(r, z=0) * r d r d \varphi$

Solved after $\mathrm{I}_{0}$, the equation results in:

$I_{0}=\frac{2 * P_{L}}{\pi * w_{0}^{2}}$

This results in the following spatial intensity distribution:

$I(r, z)=\frac{2 * P_{L}}{\pi * w_{0}^{2}} *\left(\frac{w_{0}}{w(z)}\right)^{2} * e^{\frac{\left.-2^{*}(r) 2\right)}{w(z))^{2}}}$ 
By a transformation into Cartesian coordinates and by consideration of the movement of the laser beam, $r^{2}$ can be reformulated as follows:

$r^{2}=\left(x-v^{*} t\right)^{2}+y^{2}$

Considering the intensity reduction according to the Lambert Beer law and the reflection at the boundary surface, the following equation results for the heat source:

$Q=(1-R) * \alpha * \frac{2 * P_{L}}{\pi * w(z)^{2}} * e^{-\alpha * z-\frac{-2 *\left[\left(x-v^{*}\right)^{2}+y^{2}\right]}{w(z))^{2}}}$

\subsection{Modelling of the welding process}

The thermal simulation was carried out using the FEM software Comsol Multiphysics® (Comsol Inc., Stockholm, Sweden). Here, a polycarbonate (PC) was modelled as a sample with a thickness of $2 \mathrm{~mm}$. In order to calculate the absorbed laser intensity inside the material, the optical properties were measured using the UV/VIS/NIR spectrometer Lambda 1050 (Perkin Elmer Inc., Waltham, MA). At $1940 \mathrm{~nm}$, the absorption is $15 \%$, the reflection is $9 \%$, and the transmission is $76 \%$. The sample is enclosed by a glass plate and an aluminium plate, which form the clamping device (see Fig. 1). About $85 \%$ of the laser radiation passes through the glass plate and is absorbed by the sample. The remaining radiation is reflected back into the sample by the aluminium plate. Roughness has been neglected for all components.

Since in the ideal case the temperature distribution in the plastic is symmetrical, only half of the sample was modelled to simplify matters. This shortens the calculation time. The beam properties were measured using the scanning diagnostics system Focus Monitor (Primes $\mathrm{GmbH}$, Pfungstadt, Germany) and implemented in the simulation model. The seam length is $16 \mathrm{~mm}$. In this work, the HAZ is defined as the area where the glass transition temperature of PC $\left(\mathrm{T}_{\mathrm{G}}=145^{\circ} \mathrm{C}\right)$ is exceeded. The value was taken from the literature [17].

The simulations were performed for different welding parameters in order to investigate their influence on the HAZ. For a better comparability, the line energy $\mathrm{E}$ was used. It is defined as:

$E=\frac{P}{v} *_{n}$

where $P$ is the laser power, $v$ is the feed rate and $n$ is the number of repeats. As described in Chapter 2.1, the heat conduction equation consists of a convective and a diffusive component. The Péclet number describes the ratio between the feed rate, the beam diameter and the thermal diffusivity. It is an important parameter for characterising the temperature development:

$P e ́=\frac{v^{*} w_{0}}{a}$

where $v$ is the feed rate, $w_{0}$ is the beam diameter and $a$ is the thermal diffusivity. The convective energy flow is influenced by the feed rate, whilst the diffusive term is determined by the thermal diffusivity. In case of Pé $>>1$, the convective heat flow dominates, so that the temperature distribution corresponds to the moving heat source. This is the case with high feed rates. For Pé $<<1$, the diffusive heat flux is dominant, which results in a wider temperature field in the material.

\section{Simulation results}

\subsection{Simulation of the heating performance}

In a first step, the temperature development in the interface between the upper and lower joining partners was simulated.
Fig. 1 Sample geometry of the simulation model

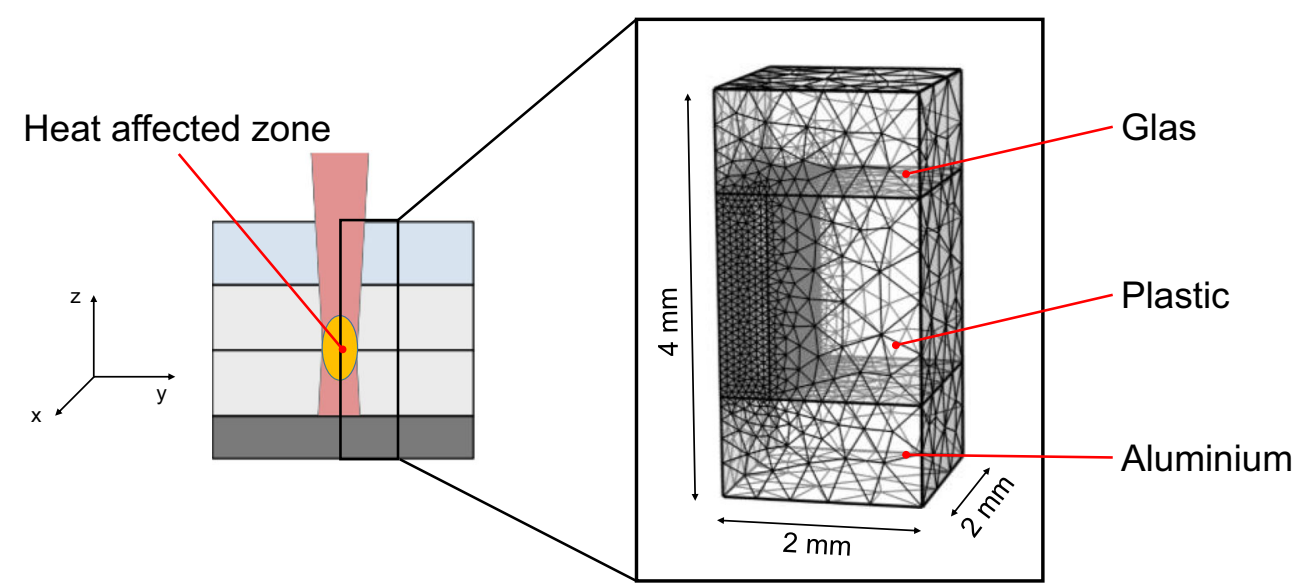


The welding parameters selected were a constant line energy of $2.5 \mathrm{~J} / \mathrm{mm}$ and a laser power of $22.5 \mathrm{~W}$. The number of passes was increased from $n=1$ to $n=150$. In order to keep the line energy constant, the feed rate must be adjusted accordingly. For $n=1$, the feed rate is therefore $9 \mathrm{~mm} / \mathrm{s}$, whilst for $n=150$, it increases to $1350 \mathrm{~mm} / \mathrm{s}$.

$E=\frac{22.5 \mathrm{~W}}{9 \frac{\mathrm{mm}}{\mathrm{s}}} * 1=\frac{22.5 \mathrm{~W}}{1350 \frac{\mathrm{mm}}{\mathrm{s}}} * 150=2.5 \mathrm{~J} / \mathrm{mm}$

For $n=1$, the highest temperature in the material is achieved with $T=291{ }^{\circ} \mathrm{C}$ (see Fig. 2). The short but strong heating of the material and the subsequent cooling process can also be seen. Overall, contour welding is characterised by a short interaction time between the laser beam and the material. For higher $n$, the welding process can be divided into $n$ heating and cooling phases. A heating phase $t_{\mathrm{i}}$ is followed by a cooling phase $t_{\mathrm{c}}$, during which heat is transferred to the aluminium and glass plates via the outer surfaces of the sample. From $n=5$, a lower maximum temperature is reached in the material due to the cooling phases. In contrast to contour welding, the interaction times are longer. The results are shown in Fig. 2.

From $n=100$, the cooling phase decreases due to the high feed rates to such an extent that a single heating phase can be assumed (tc $\approx 0$ ). Consequently, a quasisimultaneous heating of the entire seam segment takes place. This shifts the ratio of the heat flow, and the convective term can be neglected. This in turn leads to a decrease of the Péclet number.

\subsection{Simulation of the temperature distribution}

The following figure (Fig. 3) shows the temperature distribution along the cross section of the sample. The sample is located between $z=1 \mathrm{~mm}$ and $z=3 \mathrm{~mm}$, and the glass and aluminium plate is located above and below it. For $n=1$, the highest temperatures are reached inside the polymer, whereas the material temperature decreases with increasing number of passes. The glass transition temperature is therefore exceeded in a smaller range. Consequently, the reduction of the vertical expansion of the HAZ can be achieved by means of quasisimultaneous welding. For all parameters, the maximum temperature is not located in the interface, but in the upper joining partner. The HAZ is thus shifted in the direction of the upper joining partner. The reason for this is the higher thermal conductivity of aluminium compared with glass. Whilst the aluminium plate is not heated significantly, there is an increase in temperature in the glass plate. Another reason is that the laser intensity is highest on the surface of the upper joining partner.

In case of the HAZ width, the highest maximum temperature in the plastic is achieved with a value of $292{ }^{\circ} \mathrm{C}$ for $n=1$. It can be seen that the temperature gradient is significantly greater than for all other parameters. The glass transition temperature is also exceeded in a wider range. This means that the highest HAZ width is achieved during contour welding. As the number of passes increases, the maximum temperature in the polymer decreases. A reduction in the HAZ width is also achieved. However, the reduction occurs to a lesser extent compared with the HAZ height. The reason for this is that the heat in the joining zone is not dissipated via the outer surfaces. Instead, the heat flows off into the surrounding
Fig. 2 Temperature progression for different number of passes

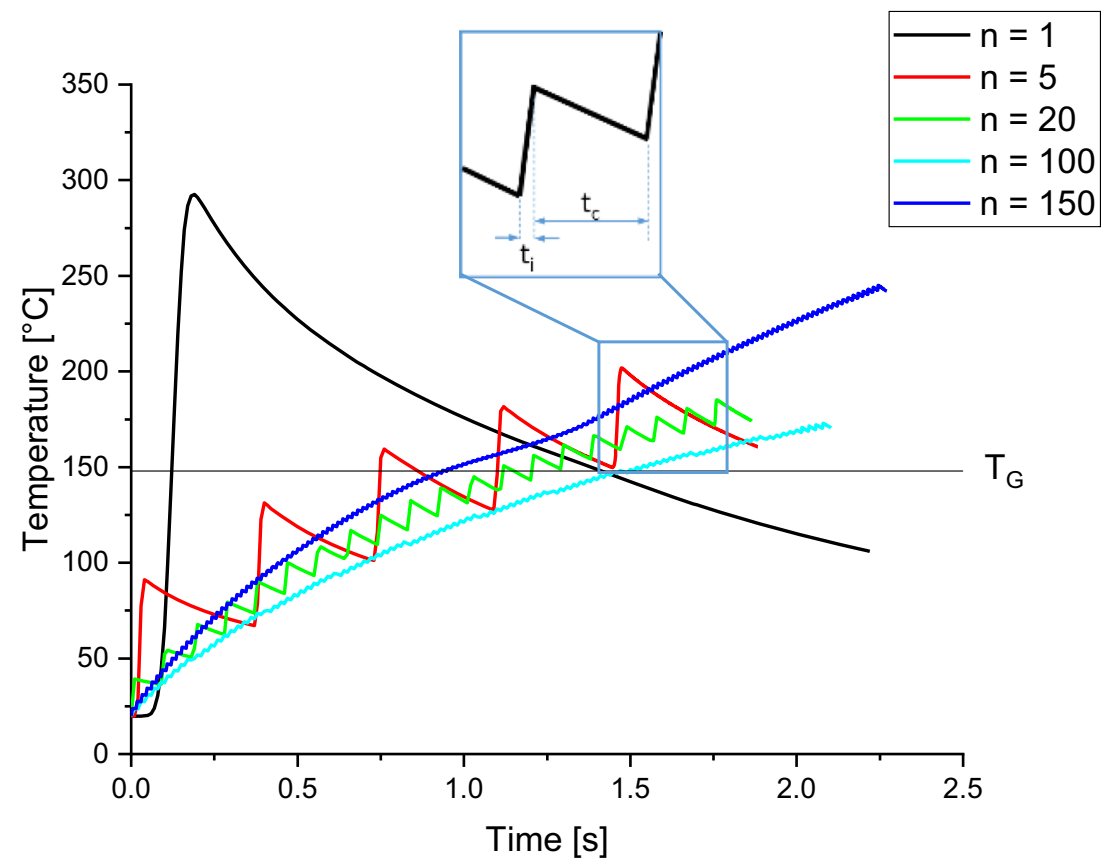




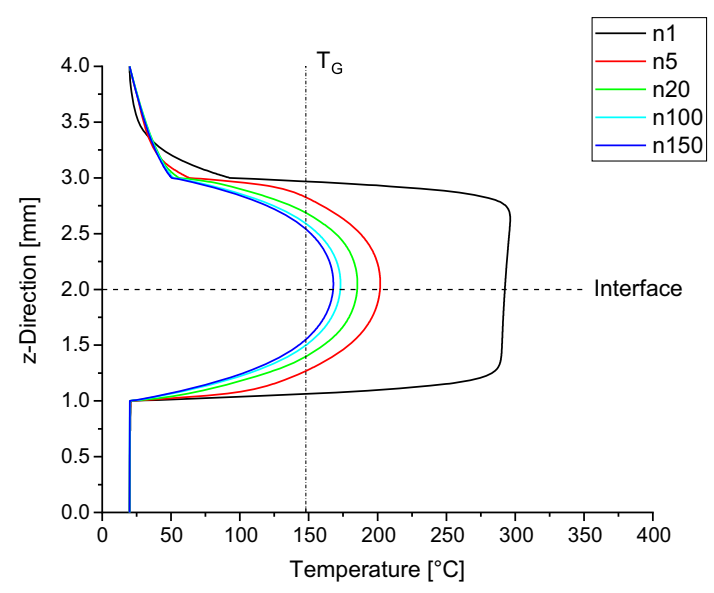

Fig. 3 Vertical (left) and horizontal temperature distribution (right)

material, which also leads to heating here. Contour welding, on the other hand, heats the surrounding material only slightly.

\subsection{Sensitivity analysis}

The results presented above show the influence of the process parameters number of passes $n$ and feed rate $v$ on the temperature distribution and the HAZ. However, the influence of the following parameters is unknown on the generation of the HAZ:

- Absorption coefficient $\alpha$

- Thermal conductivity of the polymer $\lambda_{\mathrm{PC}}$

- Thermal conductivity of the glass plate $\lambda_{\text {glass }}$

- Beam diameter $\mathrm{w}_{0}$

- Density and heat capacity $\rho * c_{P}$

In order to quantify the influence, a sensitivity analysis is carried out according to the one-at-a-time technique (OAT). Only one input variable is varied, whilst the remaining parameters remain unchanged. In total, each parameter was varied

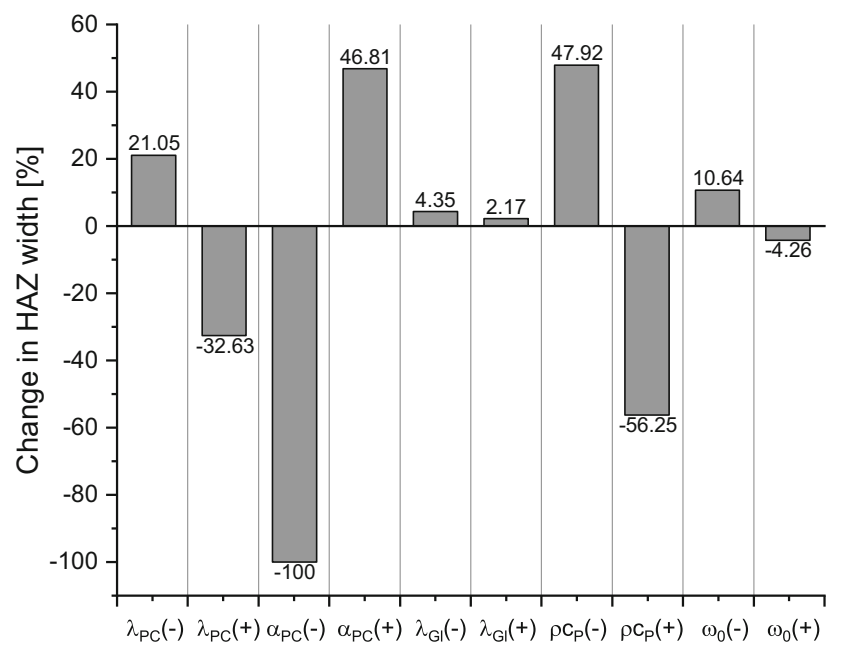

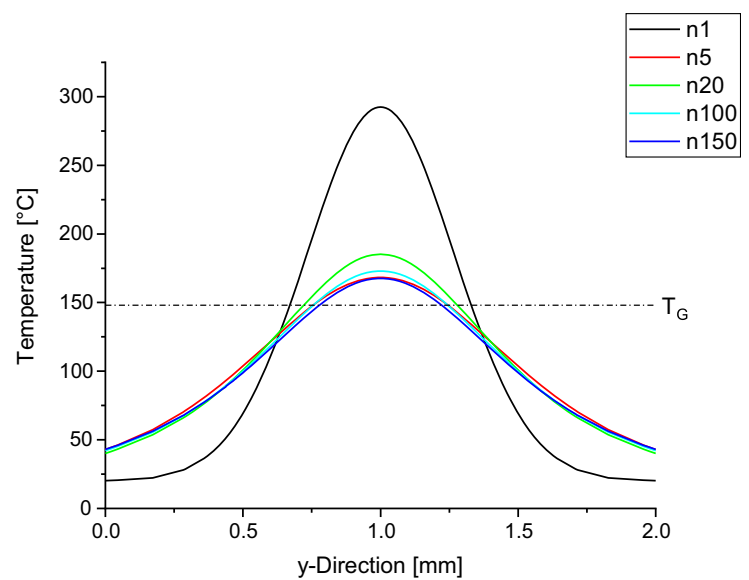

by $\pm 30 \%$, and the effect on the HAZ was investigated. The simulations were carried out for $P=2.5 \mathrm{~W}, n=20$ and $\mathrm{v}=$ $180 \mathrm{~mm} / \mathrm{s}$, so that the line energy is $E=2.5 \mathrm{~J} / \mathrm{mm}$. The results are shown in Fig. 4.

In a first step, the thermal conductivity $\lambda_{\mathrm{PC}}$ was varied. The lower the value, the stronger the effect of heat accumulation becomes and the less heat is dissipated into the surrounding material and the outer surfaces of the joining partners. With a $30 \%$ reduction of $\lambda_{\mathrm{PC}}$, the HAZ width increases by $21 \%$ and the HAZ height by $23.5 \%$. However, if $\lambda_{\mathrm{PC}}$ is reduced, more heat will flow into the environment during the welding process. In this case, the HAZ width is reduced by $32.6 \%$ and the height by $31.3 \%$. The absorption coefficient $\alpha$ determines the absorbed laser energy along the beam propagation direction.

If $\alpha$ is reduced by $30 \%$, the absorbed radiation is not sufficient to form a HAZ. As a result, the HAZ width and height are changed by $-100 \%$. An increase leads to an increase in the HAZ width and height of $48.8 \%$ and $25 \%$, respectively.

Of all investigated parameters, the thermal conductivity of the glass plate has the least influence on the formation of the

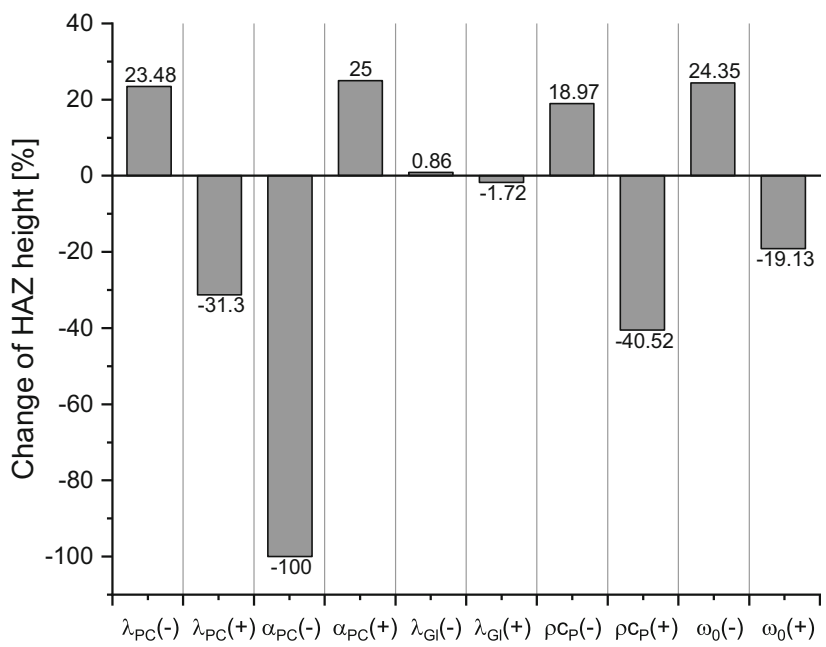

Fig. 4 Change of the HAZ width (left) and HAZ height (right) after the sensitivity analysis 

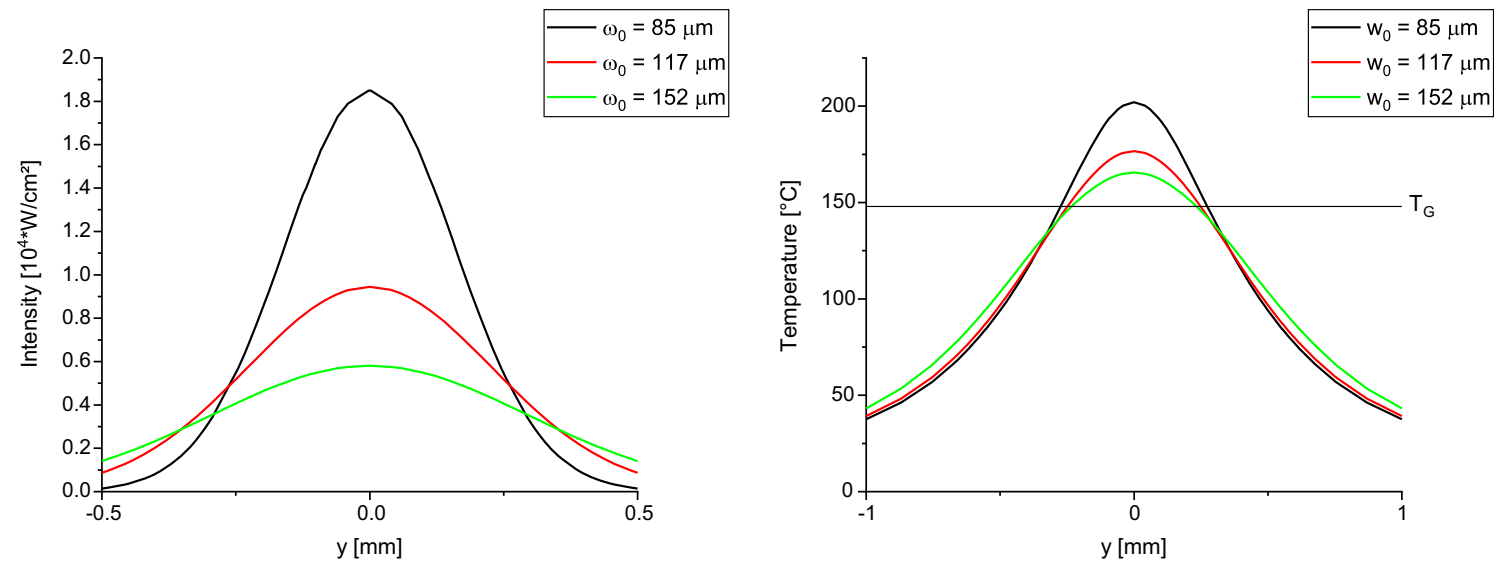

Fig. 5 The laser intensity (left) and temperature distribution (right) inside the material depending on the focal diameter

HAZ. With a reduction, the HAZ width increases by $4.3 \%$, while the HAZ height increases by $0.86 \%$. If the thermal conductivity of the glass plate is increased, the HAZ height decreases by $1.7 \%$, but the HAZ width also increases by $2.1 \%$. Due to the small amount of the change, this can be attributed to measurement errors.

In addition to the absorption coefficient, the product of the density and the heat capacity of PC also have a major influence on the HAZ. A reduction of $\rho * c_{p}$ leads to an increase of $47.9 \%$ in the HAZ width and $19 \%$ in the HAZ height. The HAZ width decreases by $56.2 \%$ with an increase of $\rho * c_{P}$, while the HAZ height decreases by $40.5 \%$.

In contour welding, the HAZ dimensions are mainly determined by the focal diameter. In the theoretical consideration of quasi-simultaneous welding, the effect is reversed. In the case of a reduction of the focal diameter, the HAZ width increases by $10.6 \%$, while the HAZ height increases by $24.3 \%$. If the focal diameter is increased, the HAZ width and height decrease by $4.2 \%$ and $19.1 \%$, respectively. In order to explain this effect, it is necessary to observe the intensity and temperature distribution perpendicular to the scanning direction.

Figure 5 on the left shows the intensity distribution in the joining zone. With a focal diameter of $85 \mu \mathrm{m}$, the maximum intensity is $1.85 * 10^{4} \mathrm{~W} / \mathrm{cm}^{2}$. If the focal diameter is increased to $152 \mu \mathrm{m}$, the maximum intensity drops to
$0.58 * 10^{4} \mathrm{~W} / \mathrm{cm}^{2}$. The intensity thus drops by approx. $66 \%$. The higher focal diameter also leads to a widening of the intensity distribution. As a result, the intensity at a distance of $\pm 0.29 \mathrm{~mm}$ is greater at a focal diameter of $152 \mu \mathrm{m}$ than at $85 \mu \mathrm{m}$. A reduction in the HAZ dimensions with a larger focal diameter is due to the fact that the threshold intensity required for melting is exceeded in a smaller range. This is also shown by the temperature distribution in Fig. 5 on the right. At $w_{0}=$ $152 \mu \mathrm{m}$, the maximum temperature in the joining zone is $165{ }^{\circ} \mathrm{C}$, whilst at $w_{0}=85 \mu \mathrm{m}$, the temperature is $201{ }^{\circ} \mathrm{C}$. Also, with a higher focal diameter, the glass transition temperature is exceeded in a smaller range.

However, it should be noted that this is only a theoretical consideration of the welding process. Whether the abovementioned effect also occurs in real welding tests must be proven in future studies.

\section{Experimental validation}

\subsection{Experimental setup}

The experimental setup is shown in Fig. 6. As testing material, PC with a thickness of $1 \mathrm{~mm}$ was used (Makrolon $®$, Covestro AG, Leverkusen, Germany). The samples were placed in a

Fig. 6 Experimental setup

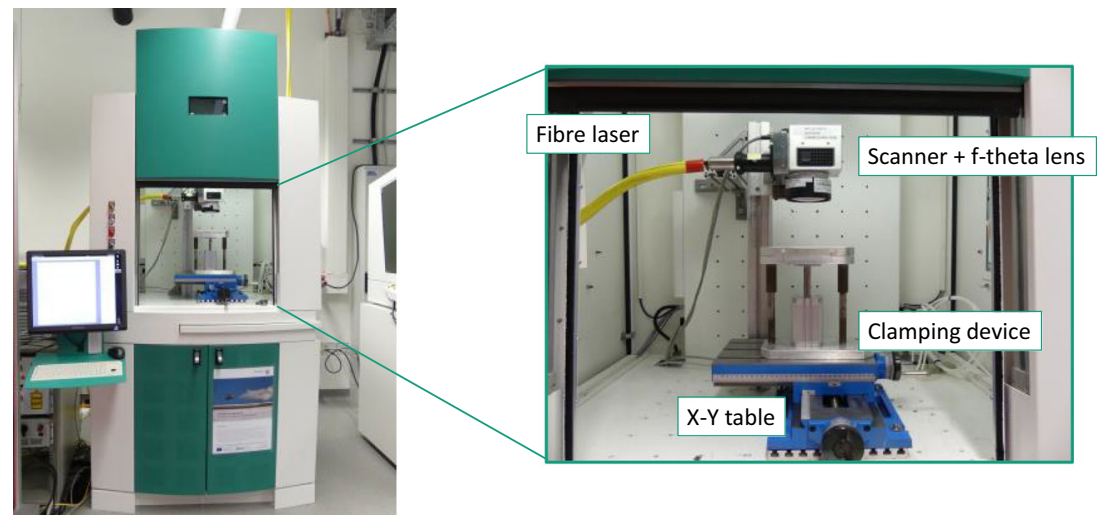


Table 1 Summary of the laser system and the beam properties

\begin{tabular}{llll}
\hline Laser system & & Beam properties \\
\hline Beam source & IPG TLR-120-1940 & Focal diameter & $116 \mu \mathrm{m}$ \\
Scanner & SCANLAB IntelliSCAN III 14 & Rayleigh length & $1.97 \mathrm{~mm}$ \\
Focal length & $100 \mathrm{~mm}$ & Angle of divergence & $1.65^{\circ}$ \\
\hline
\end{tabular}

clamping device which consists of a glass and an aluminium plate. This enables a thermal contact as well as a reproducible positioning. The samples were than irradiated using a thulium fibre laser with a maximum output power of $P=120 \mathrm{~W}$ (IPG Laser GmbH, Burbach, Germany). The laser beam was moved using the scanning system IntelliSCAN III 14 (Scanlab GmbH, Puchheim, Germany) and focused using a telecentric f-theta lens $(\mathrm{f}=100 \mathrm{~mm})$.

The welding seams were investigated using a polarisation microscope Axio Imager A2.m (Carl Zeiss Microscopy $\mathrm{GmbH}$, Jena, Germany). Here, thin sections were made of the samples perpendicular to the welding seams using a rotary microtome RM2255 (Leica Biosystems Nussloch GmbH, Nussloch, Germany). A summary of the laser system and the beam properties can be found in Table 1 .

\subsection{Comparison between experimental and simulational results}

In the following, the HAZ dimensions determined by the simulation are compared with the values from the real welding tests. Figure 7 (left) shows the height of the HAZ as a function of the number of passes. As the number of passes and feed rate increases, the diffusive energy flow dominates, and consequently more heat is dissipated via the outer surfaces of the joining partners. In case of the HAZ height, a value of $1.76 \mathrm{~mm}$ was reached for $n=5$. For $n=150$, the HAZ height was reduced to $1 \mathrm{~mm}$ which leads to a reduction of $43.4 \%$. In the simulation on the other hand, a reduction of $67.4 \%$ was

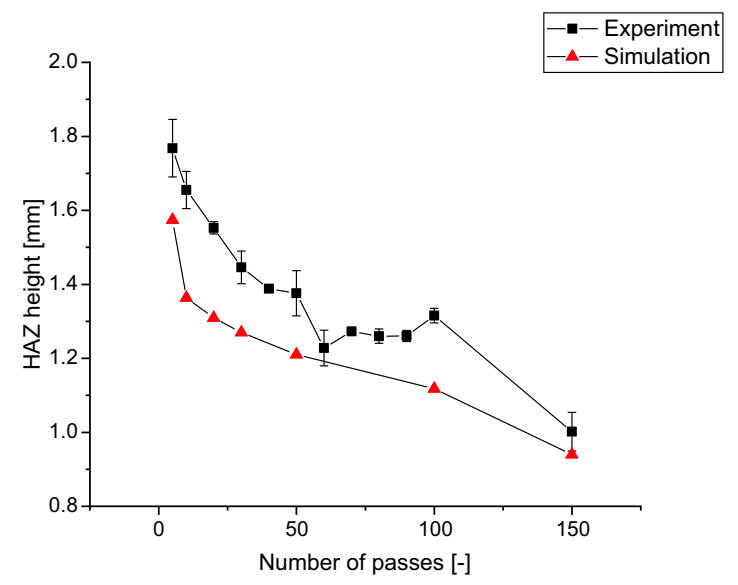

Fig. 7 Comparison between the simulation and the experimental results achieved. On average, the deviation between the simulated and the experimental HAZ height is $12.8 \%$.

A similar trend can be observed for the HAZ width. The HAZ width is $0.4 \mathrm{~mm}$ for $n=5$, whilst at $n=150$, the HAZ width is reduced to $0.3 \mathrm{~mm}$. This leads to a reduction of $33.2 \%$. The simulated results, however, show a reduction of $46.3 \%$. It can also be seen that there is a high deviation between the simulated and experimental HAZ width with a value of $48.5 \%$.

Additionally, the simulation model predicts a smaller HAZ height compared with the experimental results. On the other hand, the simulated HAZ width is significantly higher than the real HAZ width.

A possible reason for the deviations is that simplifications in the simulation model were assumed to reduce the computing time. For example, a perfect thermal contact between both joining partners was assumed, and the roughness was therefore neglected. An ideal reflection of the laser radiation at the glass plate was also assumed. Another reason can be the influence of the interaction time between laser radiation and plastic on the melting temperature.

In a previous work by Boglea, it was indicated that the melting temperature is shifted towards higher temperatures with shorter interaction times [18]. For example, the melting temperature of a polymer is usually obtained by differential scanning calorimetry or thermogravimetric analysis measurements. Here, the heating rates are only $10-30^{\circ} \mathrm{C} / \mathrm{min}$. In laser transmission welding, the heating rates can reach up to $150{ }^{\circ} \mathrm{C} / \mathrm{ms}$. In this work on the other hand, a possible increase

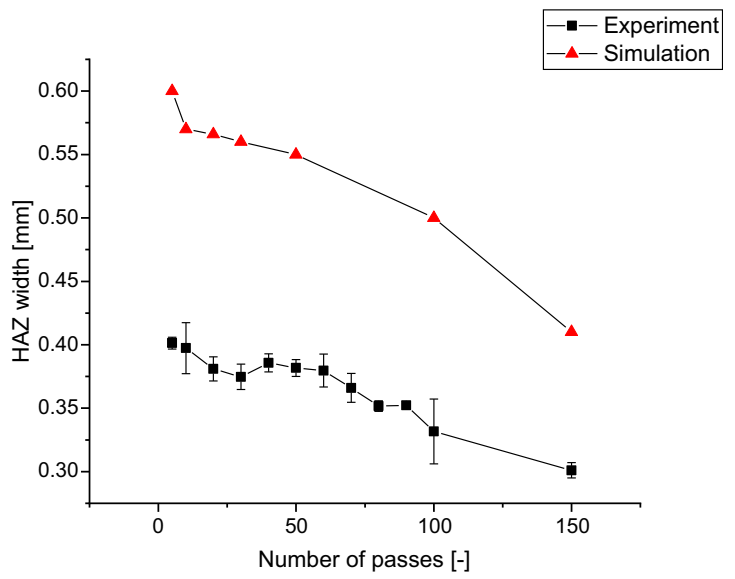


of the melting temperature due to shorter interaction times was not considered.

\section{Conclusion}

In the present work, a model for the calculation of the temperature distribution during absorber-free quasi-simultaneous welding of plastics with a wavelength of $1940 \mathrm{~nm}$ was presented. It could be shown that the diffusive heat transport dominates in quasi-simultaneous welding. With higher feed rate and number of passes, the heat dissipation allows a reduction of the HAZ in the vertical and horizontal direction in comparison to contour welding. In order to quantify the influence of the beam and material properties on the HAZ geometries, a sensitivity analysis was performed. The simulation model is able to predict the HAZ height in good agreement to the experimental results. Here, the average deviation is $12.8 \%$. However, there is a high deviation between the simulated and experimental HAZ with an average deviation of $48.5 \%$. The reason for the high deviations will be investigated in future works. Possible reasons are simplifications, which were assumed in the simulation model. Another point is the influence of the interaction time between laser radiation and material on the melting temperature of the polymer. Past studies indicated that the melting temperature increases with decreasing interaction time.

Acknowledgements Open Access funding provided by Projekt DEAL.

Funding information This project is co-funded by the European Regional Development Fund and the German federal state of North Rhine-Westphalia (EFRE-0800726).

Open Access This article is licensed under a Creative Commons Attribution 4.0 International License, which permits use, sharing, adaptation, distribution and reproduction in any medium or format, as long as you give appropriate credit to the original author(s) and the source, provide a link to the Creative Commons licence, and indicate if changes were made. The images or other third party material in this article are included in the article's Creative Commons licence, unless indicated otherwise in a credit line to the material. If material is not included in the article's Creative Commons licence and your intended use is not permitted by statutory regulation or exceeds the permitted use, you will need to obtain permission directly from the copyright holder. To view a copy of this licence, visit http://creativecommons.org/licenses/by/4.0/.

\section{References}

1. Aden M, Mamuschkin V, Olowinsky A (2015) Influence of carbon black and indium tin oxide absorber particles on laser transmission welding. Opt Laser Technol 69:87-91. https://doi.org/10.1016/j. optlastec.2014.12.015

2. Haberstroh E, Hoffmann WM, Poprawe R, Sari F (2006) Laser transmission joining in microtechnology. Microsyst Technol 12: 632-639. https://doi.org/10.1007/s00542-006-0096-0

3. Mamuschkin V, Haeusler A, Engelmann C, Olowinsky A, Aehling $H$ (2017) Enabling pyrometry in absorber-free laser transmission welding through pulse irradiation. J Laser Appl 29:1-6. https://doi. org/10.2351/1.4983515

4. Becker F, Potente H (2002) A step towards understanding the heating phase of laser transmission welding of polymers. Polym Eng Sci 42:365-374. https://doi.org/10.1002/pen.10954

5. Acherjee B, Kuar AS, Mitra S, Misra D (2012) Effect of carbon black on temperature field and weld profile during laser transmission welding of polymers. A FEM study. Optics Laser Technol 44: 514-521. https://doi.org/10.1016/j.optlastec.2011.08.008

6. Mamuschkin V, Engelmann C, Olowinsky A (2016) Improvement of energy deposition in absorber-free laser welding through quasisimultaneous irradiation. Phys Procedia 83:472-482. https://doi. org/10.1016/j.phpro.2016.08.049

7. Jones I (2002) Laser welding for plastic components. Assem Autom 22:129-135. https://doi.org/10.1108/01445150210697429

8. Brosda M, Olowinsky A, Pelzer A (2016) Laser encapsulation of organic electronics with adapted diode lasers in flexible production processes. Phys Procedia 83:218-224. https://doi.org/10.1016/j. phpro.2016.08.013

9. Mamuschkin V, Aden M, Olowinsky A (2019) Investigations on the interplay between focusing and absorption in absorber-free laser transmission welding of plastics. Lasers Manuf Mater Process 6: 113-125. https://doi.org/10.1007/s40516-019-00083-1

10. Chen M, Zak G, Bates PJ (2011) Effect of carbon black on light transmission in laser welding of thermoplastics. J Mater Process Technol 211:43-47

11. Kreimeier Sooriyapiragasam S, Hopmann C (2016) Modeling the heating process during the laser transmission welding of thermoplastics and calculation of the resulting stress distribution. Weld World 60:777-791

12. Aden M, Mamuschkin V, Olowinsky A, Glaser S (2014) Influence of titanium dioxide pigments on the optical properties of polycarbonate and polypropylene for diode laser wavelengths. J Appl Polymer Sci: 131

13. Xu XF, Parkinson A, Bates PJ, Zak G (2015) Effect of part thickness, glass fiber and crystallinity on light scattering during laser transmission welding of thermoplastics. Opt Laser Technol 75: 123-131

14. Zak G, Mayboudi L, Chen M, Bates PJ, Birk M (2010) Weld line transverse energy density distribution measurement in laser transmission welding of thermoplastics. J Mater Process Technol 210: 24-31

15. Chen M, Zak G, Bates PJ (2013) Descritpion of transmitted energy during laser transmission. Weld World 57:171-178

16. Nguyen N P, Brosda M, Olowinsky A, Gillner A (2019) Absorberfree quasi-simultaneous laser welding of transparent microfluidic devices. Proceedings of LAMP2019

17. Covestro (2020) Makrolon® 2205 Technical Datasheet

18. Boglea A (2013) Laser transmission welding of thermoplastics using local laser beam modulation. Dissertation, RWTH Aachen University

Publisher's note Springer Nature remains neutral with regard to jurisdictional claims in published maps and institutional affiliations. 